\title{
Detecting word endings in an unknown script
}

A Sequence Analysis, examinded on a digitalized corpus of Linear Elamite, allows for a certain insight into the morphosyntax of the underlying language, even if the phonetic values of most of the 99 signs are not known. By programming a so-called "repetitions-with-omissions" Filter, we were able to detect verbal and nominal suffixes. This combined with the knowledge about some single sound values, we now can show that the language behind must be Elamite or some idiom closely related to it. 